\title{
Human Factor Ergonomics Considerations For A Glucose Sensor Device
}

\author{
Soo Li Choong(sl.choong@mimos.my)', Muhamad Ai’dil Abd Rahman (aidil.rahman@mimos.my)1, \\ Khairul Nazri Abd Wahib (nazri.wahib@mimos.my) ${ }^{1}$
}

${ }^{1}$ Department of Appearance Design \& Mechanical Modelling, MIMOS Semiconductor (M) Sdn. Bhd., Kuala Lumpur, Malaysia, 57000

\begin{abstract}
This paper presents the methodologies and the systematic approaches of human factor ergonomics considerations in designing a Glucose Sensor device. The Glucose Sensor device is a non-invasive version of glucose sensor utilizing NIR (Near Infra-Red Spectroscopy) for medical application that provides diabetes screening as an early symptom in diagnostic. Contextual inquiry (CI) was conducted at the early stage to gather all the users' inputs and feedback and KJ Analysis was applied to prioritize the voice of customer. Hence, the customer needs could be identified as our design requirements. This study encompasses the ease of use and the usability of the Glucose Sensor device on the two main product features which are thumb module design and LCD placement. Prototypes had been developed to assess the design effectiveness and the discussion will be carried out at the end of this paper.
\end{abstract}

Keywords: Glucose Sensor device, Human Factor Ergonomics (HFE) considerations, Contextual inquiry, Thumb module design, LCD placement, ESK-JES Joint Session

\section{Introduction}

The Glucose Sensor device is a non-invasive version of glucose sensor utilizing NIR (Near Infra-Red Spectroscopy) for medical application that provides diabetes screening as an early symptom in diagnostic. It can provide glucose measurements painlessly, without a blood sample or finger pricks, within a few seconds. Current practice of glucose sensor requires a blood draw through finger pricks for each test, which causes pain and inconvenience. Besides that, each test also requires a new test-strip, contributing to the recurring cost of such a device. Therefore, the Glucose Sensor device is developed that aims to overcome the issues addressed.

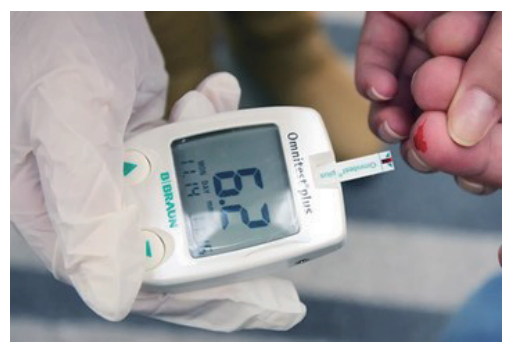

Figure 1. One of the current practices of glucose sensor (Source: http://mu-varna.bg/EN/Pages/glukozni_senzori.aspx)

\section{User Requirements}

There are some rules and requirements to comply when designing a device for medical use. In 2000, the FDA's Center for Devices and Radiological Health (CDRH) published its guidance entitled Medical Device Use Safety: Incorporating Human Factors Engineering in Risk Management relating human factors, ergonomics, and usability methods directly to the process of risk management in device design (Kaye \& Crowley, 2000). By applying HFE, it can minimize use-related hazards and risks and users can use the device safely and effectively (Sawyer et al., 1996). Besides the necessary requirements and standard rules, the users' needs are also important to the Glucose Sensor device design. Knowing that for diabetic patients, they have decreased in tactile sensation, vision loss, hearing loss, limb loss, and decreased mobility (ANSI/AAMI HE75, 2010). Hence, it is important to understand the product end users and design it that meet specifications and customer needs. Initial product specifications can be generated through collecting voice of customers, and conducting competitive benchmarking and market analysis against successful products in the market. 


\subsection{Contextual Inquiry}

At the preliminary stage of the design cycle, initial design specifications for the product were established by translating the user needs into design requirements. This will ensure the design meets customers' needs and expectations. User needs, in other word, Voice of Customer (VOC) were gathered through Contextual inquiry (CI). CI is a human factors research method that combines the questioning and observation of users within the environment of use. It has its roots in ethnography and aims to collect contextual information by observing people in their work or living environment (Martin \& Crowe, 2010). Figure 2 illustrates the actual working condition when CI was conducted at the targeted hospital.
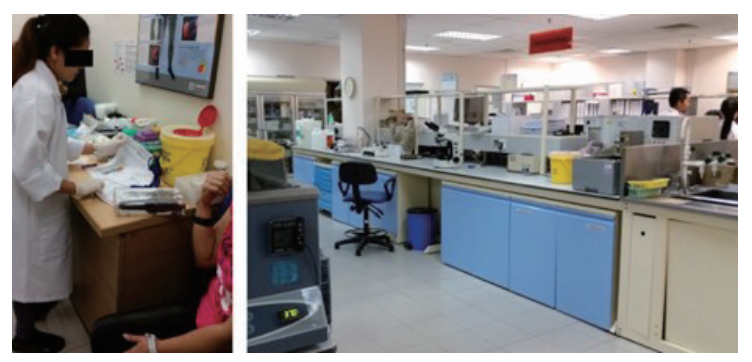

Figure 2. Actual working condition in the targeted hospital.

The VOC were then prioritized using $\mathrm{KJ}$ analysis as illustrated in Figure 3. The analysis result showed that the category of Good Functionality is the most important design criteria for the Glucose Sensor Device with the highest scoring, followed by Good Appearance and Easy Maintenance. The design criteria of Good Functionality and then translated into technical design parameters by using House of Quality (HOD). The method of HOD will not be discussed here.

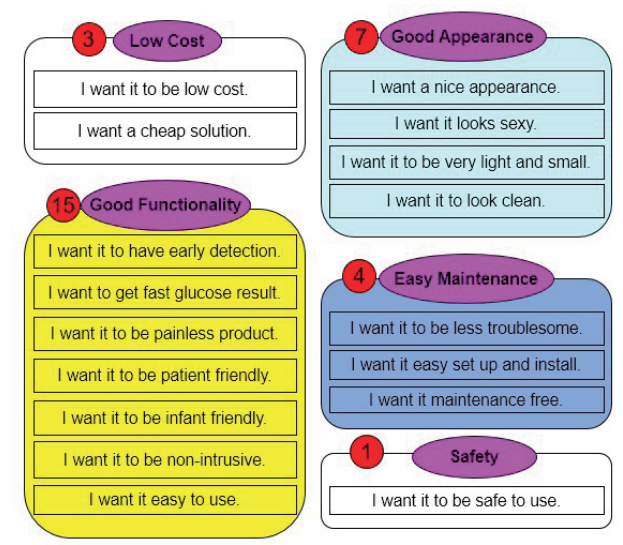

Figure 3. KJ Analysis for Glucose Sensor device.
From the analysis, two main product features were identified: thumb module and LCD placement. These two items were the main features that associated to the functionality of the device.

\subsection{Benchmarking}

After the design requirements had been identified using $\mathrm{KJ}$ analysis, design benchmarking was performed as well to benchmark against similar existing products in the current market. The aims were to understand market trends, compare features and faults in existing product in the market, at the same time finding opportunities for improvement in our own product. Figure 4 illustrates the benchmarking on some glucose sensor devices that are in the market.

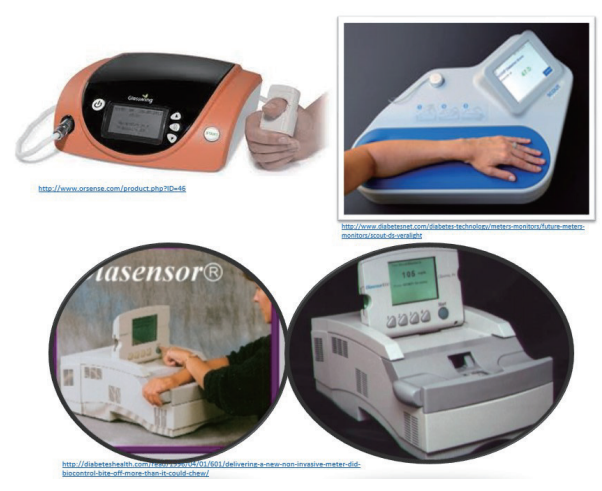

Figure 4. Benchmarking on non-invasive glucose sensor devices.

\section{Design}

At the design stage, several concepts were generated and prototypes were evaluated and reviewed. This design process is an iterative process until the results is satisfactory, and all the technical requirements are met.

\subsection{Thumb Module}

Due to the non-invasive application, the Glucose Sensor device is designed with a thumb module that allows users to place their thumb in a designated enclosure and the device will take the reading from the thumb as shown in Figure 5. Different users have different sizes of the thumb, 
hence designing a thumb module that fits all the sizes is crucial to ensure satisfaction of the users and efficiency of data collecting of the device. Anthropometry and biomechanics of human was referred and considered in this design study.

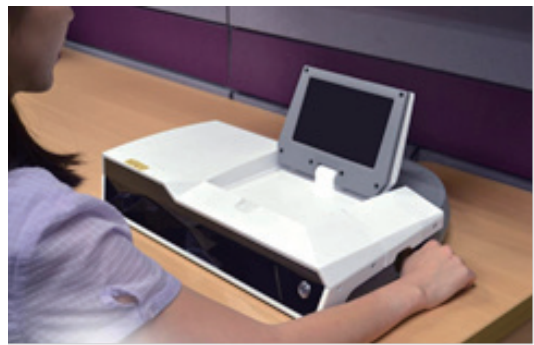

Figure 5. Thumb insertion into the thumb module for data reading.

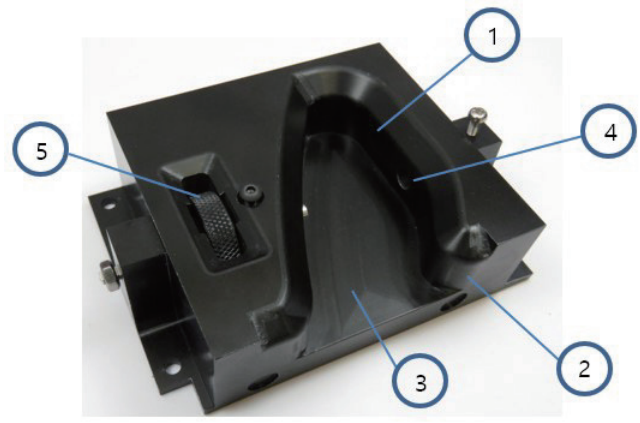

Figure 6. The thumb module for the Glucose Sensor device.

Figure 6 demonstrates the thumb module design. Each design feature of the thumb module had incorporated human factor ergonomics considerations as below:

(1) Thumb profile based on anthropometry data to fit general thumb sizes,

(2) Big radius to avoid sharp edges and hazardous to the users,

(3) Rubbery base (biocompatibility compliance) to provide comfort to the users,

(4) Slight protrusion on the surface to provide indication to the users for fast thumb alignment,

(5) Single-handed adjustable feature for the ease of use.

\subsection{LCD Display}

There is a LCD display on the Glucose Sensor Device for information display and data entry purposes. The location of the LCD display determines the viewing angle and the sitting postures of the users, in this case both medical officers and patients.

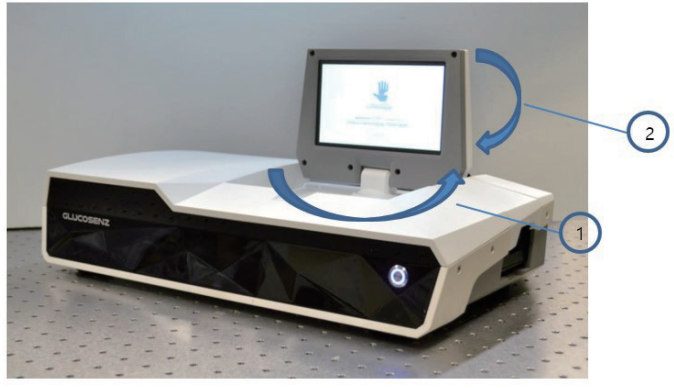

Figure 7. Glucose Sensor device with LCD flipped up.

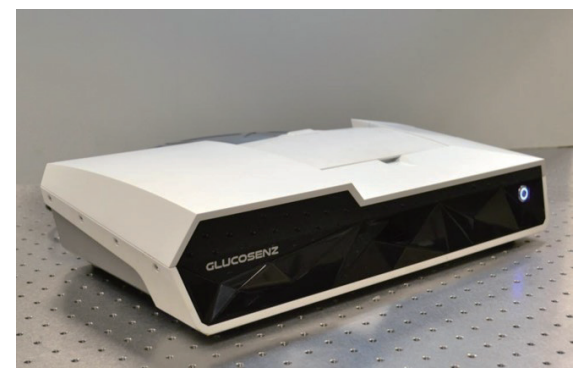

Figure 8. Glucose Sensor device with LCD flipped down.

Figure 7 demonstrates the LCD display flipped up (when in-use) while figure 8 demonstrates the LCD display flipped down (when not in-use) condition. Below are the ergonomic considerations for the LCD design:

(1) The screen can be rotated at $180^{\circ}$ for viewing angle adjustment,

(2) The screen can be tilted up to meet $15^{\circ}$ optimum view angle from normal eye sight.

With the final location of the LCD, both medical officers and patients could view the screen easily as illustrated in Figure 9 below.

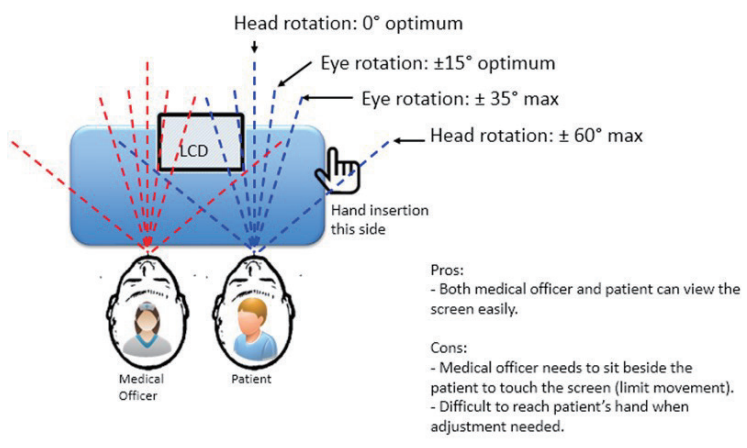

Figure 9. Study on the location of the LCD display for Glucose Sensor device. 


\section{Discussion}

In some cases, a design is not able to fulfil all the desired requirements, this is the reason why $\mathrm{KJ}$ analysis is needed to prioritize the requirements. However, in this case, some of the HFE design considerations were compromised due to the technology limitation. For example, in the ergonomic point of view, the ergonomic posture for forearm is in neutral rotation, which the arm is not twisted as shown on Figure 10.

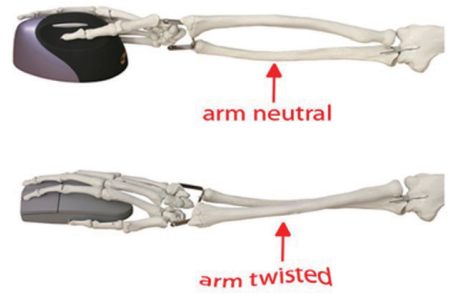

Figure 10. Ergonomic posture for forearm (Source: www.slideshare.net/khalednagib/back-forearm)

The thumb module design that met neutral arm posture during data collecting was proposed initially, however, the proposal was dropped due to the infrared light needs to be in straight condition and the bending radius must within the specific range. Neutral arm posture thumb module design was not able to meet these requirements.

Other than that, there were some findings after evaluations done on the prototypes. The recess for thumb that was initially designed for thumb guiding and user comfort was removed due to the technology limitation too. The data reading was inaccurate when there was a gap in between the light probe and the measurement object. The surface must be flat for the calibration tool to be in contact with the infrared light probe during calibration. Figure 11 shows the design changed from thumb recess to flat surface as required.
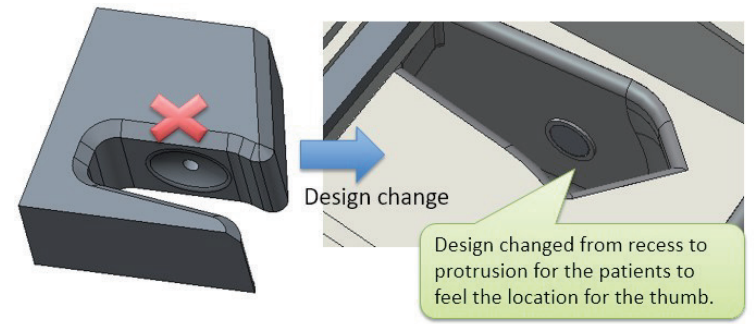

Figure 11. Design changed due to technology limitation.

\section{Conclusion}

The complexity of modelling a Glucose Sensor device has become significant when the ideal designs had compromised with the technology limitation. However, by applying the HFE approaches, users' requirements could be identified systematically and enables the engineers to design a product that is usable and effective.

\section{Acknowledgements}

We gratefully acknowledge the important contribution and guidance provided by our company internal staffs from each department who offered their great support to help us in completing this study. We would also like to show our gratitude to the external party especially the nurses, doctors and office staffs from the Sunway Medical Centre who have always been very responsive in providing necessary information. In addition, we would also like to extend our appreciation to the ACED organizer for giving us this opportunity to share our hard work here at international platform. Thank you.

\section{References}

Kaye, R. and Crowley, J., Medical Device Use-Safety: Incorporating Human Factors Engineering into Risk Management, Center for Device and Radiological Health, Silver Spring, 2000.

Sawyer, D., Aziz, K. J., Backinger, C. L., Beers, E. T., Lowery, A., Skyes, S. M., Thomas, A. and Trautman, K. A., An Introduction to Human Factors in Medical Devices, Center for Devices and Radiological Health, U.S., 1996.

Martin, J. L. and Crowe, J. A., Contextual Inquiry for Medical Device Development: A Case Study, The University of Nottingham, UK, 2010.

ANSI/AAMI HE75, Human Factors Engineering-Design of Medical Devices, Association for the Advancement of Medical Instrumentation, VA, 2010.

Peebles, L. and Norris, B., The Handbook of Adult Anthropometric and Strength Measurements-Data for Design Safety, University of Nottingham, Nottingham. 